\title{
新型特窗酸肼基衍生物的合成、杀菌及杀虫活性
}

\author{
赵宇关爱莹李婉祯王卫伟刘金否砳王明安* \\ (中国农业大学应用化学系 北京 100193)
}

\begin{abstract}
摘要 设计合成了一系列新型 3-乙酰基-4-肼基-5,5-二取代特窗酸和(E/Z)-3-(1-肼基亚乙基)-5,5-二取代 2,4-呋喃二酮衍 生物，意外得到 4 个 5-甲基吡唑-4-羧酸甲酯，它们的结构经过 HR-ESI-MS, ${ }^{1} \mathrm{H} N M R,{ }^{13} \mathrm{C}$ NMR 和 X 射线衍射的表征. 这 些肼基与氨基衍生物的生物活性测试结果表明，部分化合物显示出对测试植物病原菌中等至优异的杀菌活性. 如在 $400 \mu \mathrm{g} / \mathrm{mL}$ 浓度时化合物 5G, 5H, 5I 和 $\mathbf{5 i}$ 对炭疽病菌有 $100 \%$ 的活体抑制活性，化合物 5G 对霜霉病菌、白粉病菌和锈 病病菌的活体抑制活性均为 $100 \%$. 在 $600 \mu \mathrm{g} / \mathrm{mL}$ 浓度时化合物 $\mathbf{5 b}, \mathbf{5 E}$ 和 $6 \mathrm{~F}$ 对小菜蛾, 化合物 $6 \mathrm{~A}, \mathbf{6 g}$ 和 $6 \mathrm{H}$ 对桃蚜以及 化合物 $6 \mathbf{b}$ 对朱砂叶螨的死亡率均为 100\%. 5,5-螺环己基可以显著提高特窗酸衍生物的杀菌活性，取代肼基的引入可以 获得更高的杀虫杀螨活性. 化合物 $\mathbf{5 G}$ 和 $\mathbf{5 i}$ 是最有潜力作为研发新型杀菌剂进行结构修饰的先导化合物.
\end{abstract}

关键词 肼衍生物；螺环己基特窗酸；杀菌活性；杀虫活性

\section{Synthesis, Fungicidal and Insecticidal Activity of Novel Tetronic Acid Hydrazinyl Derivatives}

\author{
Zhao, Yu Guan, Aiying Li, Wanzhen Wang, Weiwei Liu, Xinlei Wang, Ming'an* \\ (Department of Applied Chemistry, China Agricultural University, Beijing 100193)
}

\begin{abstract}
A series of novel 3-acetyl-4-hydrazinyl-5,5-disubstitutedtetronic acid and (E/Z)-3-(1-hydrazinylethyli-dene)-5,5disubstitutedfuran-2,4-dione derivatives were designed and synthesized, and four methyl 5-methyl pyrazole-4-carboxylate were obtained unexpectedly. Their structures were confirmed by high-resolution mass spectrum (HR-ESI-MS), ${ }^{1} \mathrm{H}$ NMR, ${ }^{13} \mathrm{C}$ NMR spectral data and X-ray diffraction. The bioassay results of the hydrazinyl compounds along with the amino analogues indicated that some compounds exhibited moderate to excellent fungicidal activities against phytopathagens. For example, compounds 5G, 5H, 5I and 5i showed $100 \%$ in vivo control efficacy against Colletotrichum lagenarium, and compound 5G also exhibited $100 \%$ in vivo control efficacy against Erysiphe graminis, Puccinia polysora and Colletotrichum lagenarium at $400 \mu \mathrm{g} / \mathrm{mL}$. Compounds $\mathbf{5 b}, \mathbf{5 E}$ and $\mathbf{6 F}$ showed $100 \%$ mortality against Plutella xylostella, compounds $\mathbf{6 A}, \mathbf{6 g}$ and $\mathbf{6 H}$ exhibited $100 \%$ mortality against Myzus persicae, and compound $\mathbf{6 b}$ showed 100\% mortality against Tetranychus cinnabarinus at 600 $\mu \mathrm{g} / \mathrm{mL}$. The 5,5-spiro cyclohexyl moiety significantly improved the fungicidal activity of the tetronic acid derivatives, and the introduction of the substituted hydrazino group to the furan-2,4-dione skeleton led to higher insecticidal and acaricidal activities. 5G and 5i were found to have the most potential to be further modified for searching new fungicide as the lead compounds. Keywords hydrazine derivatives; spirocyclohexyl tetronic acid; fungicidal activity; insecticidal activity
\end{abstract}

\section{Introduction}

Tenuazonic acid, tetronic and tetramic acid, existing as furan-2,4-dione and its analogues, have attracted enormous attention in the pharmaceutical and agrochemical fields. The different substituents at various positions of them could give a large variety of functional molecules which having diverse bioactivities, such as antitumor, antioxidant, anti-HIV, anti-inflammatory and antiepileptic activities. ${ }^{[1]}$
Moreover, some natural furan-2,4-dione derivatives are the lead compounds that showed excellent antifungal and insecticidal activities against a broad range of phytopathagens and insects. For example, botryolide-E, isolated from Botryotrichum sp. (NRRL 38180), showed potent fungicidal activity against several phytopathagens at the concentration of $25 \mu \mathrm{g} / \mathrm{mL} .^{[2]}$ Pyranonigrin A, obtained from spongederived fungus Aspergillus niger, was found to effectively inhibit the growth of the larval of the plant insect Spodop-

* Corresponding author. E-mail: wangma@cau.edu.cn

Received November 30, 2018; revised January 28, 2019; published online February 22, 2019.

Project supported by the National Natural Science Foundation of China (Nos. 21772229, 21172254).

国家自然科学基金(Nos. 21772229, 21172254)资助项目. 
tera littoralis $^{[3]}$ (Figure 1). Recently, great efforts have been made to develop new tetronic acid derivatives for screening highly effective fungicides and insecticides by introducing the different substituents at C-3, C-4, and C-5 positions of furan-2,4-dione. The previous modification at $\mathrm{C}-5$ position with alkyl groups showed positive effect for improving the bioactivity. ${ }^{[4,5]}$ Especially, the introduction of 5,5-spirocyclo moieties at the skeleton of tetronic acid led to the discovery of new classes of insecticides such as spirodiclofen, spiromesifen and spirotetramat (Figure 1), which indicated that the 5,5-spirotetramic and 5,5-spirotetronic acid derivatives changed the mode of action with the greatly improved activities. ${ }^{[6-8]}$<smiles>CC(=O)OC(C)CC(O)C1C=CC(=O)O1</smiles>

Botryolide-E<smiles>C/C=C/c1oc2c(c(=O)c1O)C(=O)N[C@H]2O</smiles>

Pyranonigrin A

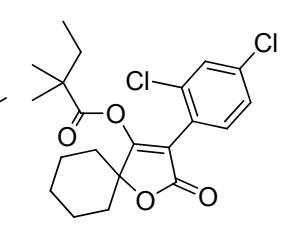

Spirodiclofen
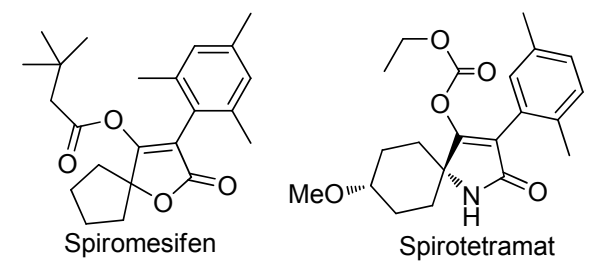

Figure 1 Representative natural bioactive tetronic and tetramic acid derivatives and insecticides

In the past decades, the synthesis and biological activity evaluation of 5-akyltetronic acid derivatives have also attracted enormous attentions. ${ }^{[9,10]}$ At the same time, various imidazolinone-containing compounds were found in nature or synthesized, and they displayed a wide range of biological activities. For example, fenamidone had excellent antimicrobial and fungicidal activities as a commercial systemic foliar fungicide. ${ }^{[11,12]}$ The 5-(4,5,5-trimethylbutenolide-3-ethylidene)-2-aminoimidazolin-4-one derivatives have been successfully prepared, and it was found that these compounds showed significant fungicidal activities against several important agricultural phytopathogens in our laboratory, ${ }^{[13]}$ and the further optimization by 5,5-spirocycles and 4-phenyl had significantly improved their fungicidal activities. ${ }^{[14-16]}$ However, when we tried to replace 4-methyl or 4-phenyl with 4-amino groups, an unexpected Michael addition-elimination cascade reaction took place between 4-methoxytetronic acid and amines to yield 4-amino substituted tetronic acid derivatives. Hence, a group of novel 5,5-dimethyl and 5,5-spirocyclohexyl amino-containing tetronic acid derivatives at the side chain of C-3 and C-4 were synthesized, however their bioactivities have not been evaluated yet. ${ }^{[17]}$ To the best of our knowledge, hydrazine as well as its hydrazone analogues played a critical role in pharmaceutical and agrochemical research. These derivatives exhibited broad bioactivities such as antimicrobial, antiviral, antiprotozoal, antitumoral, anticonvulsant, insecticidal, herbicidal and fungicidal activity. ${ }^{[18-23]}$ In the agrochemical field, RH 5849, RH 0345 and fufenozide were found to be very effective nonsteroidal ecdysone agonists on larval Lepidoptera as insecticides ${ }^{[24-27]}$ (Figure 2). Therefore, the substituted hydrazinyl groups may be the potential pharmacophore to further improve their bioactivity of the tetronic acid derivatives. Considering the structure and reaction similarity of hydrazine and amine, the substituted hydrazinyl groups were designed to introduce at C-3 and C-4 positions of 5,5-dimethyl and 5,5-spirocyclohexyl tetronic acid (Scheme 1), and to investigate their influences on the biological activities in this report. The synthetic routes of title compounds were shown in Scheme 2, the key intermediates $\mathbf{3 a}, \mathbf{3 b}, \mathbf{4 a}$ and $\mathbf{4 b}$ were prepared following the procedures in the previous paper. ${ }^{[17]}$<smiles>[R9]OS(=O)(=O)c1ccc2c(c1C)OC(C)C2</smiles>

Figure 2 Typical representative biological activity hydrazine derivatives

\section{Results and discussion}

The general synthetic routes for the target hydrazinyl derivatives were depicted in Scheme 2. The intermediates $\mathbf{1 a} \sim \mathbf{4 b}$ were prepared according to the procedures re-

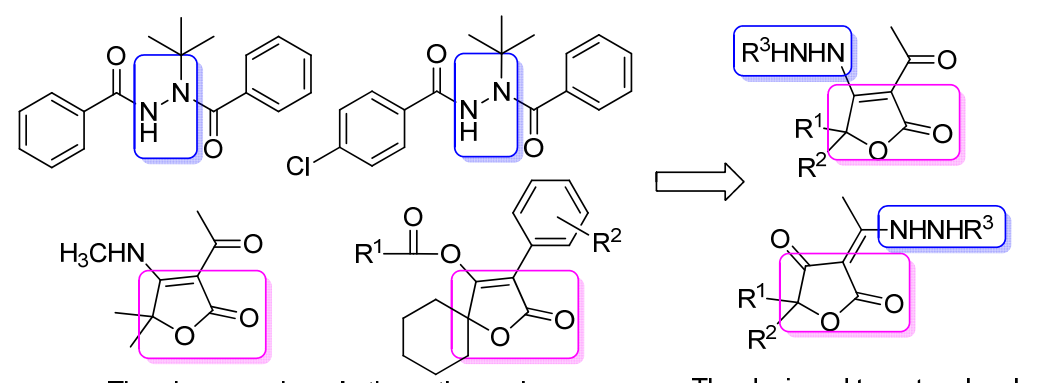

The phamacophore in the active molecues

The designed target molecules

Scheme 1 Designed novel hydrazine derivatives of 5,5-dimethyl and 5,5-spirocyclohexyl tetronic acid 


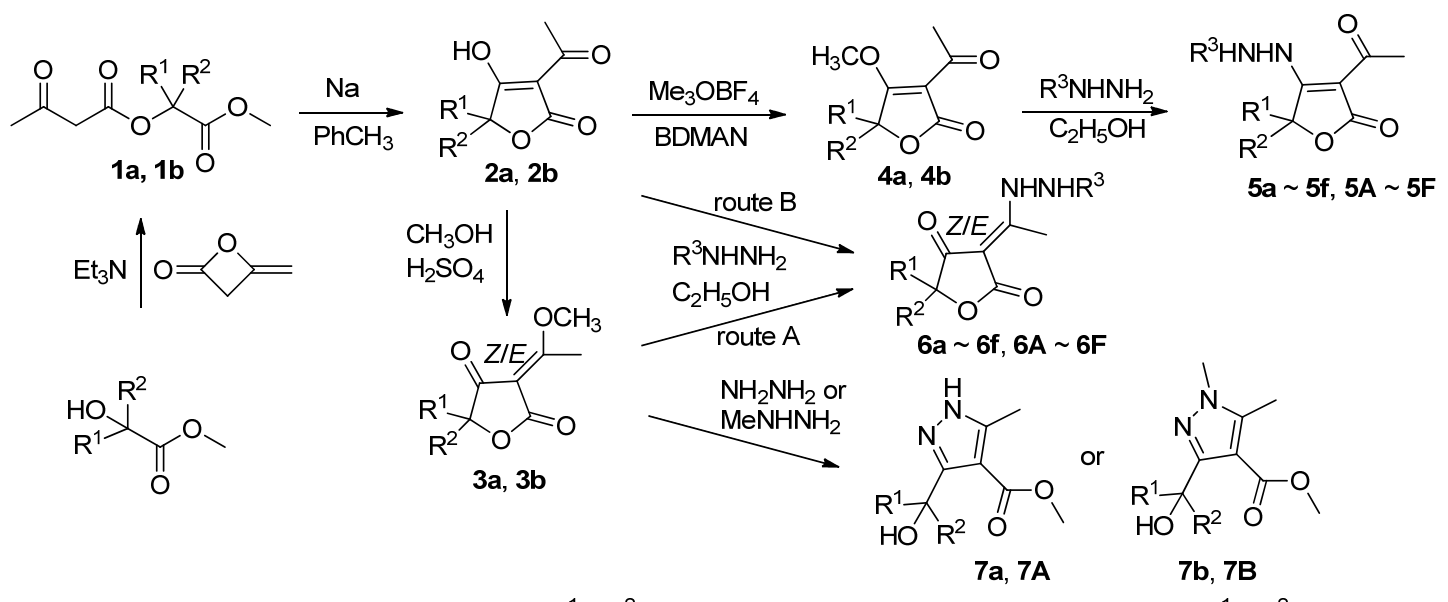

1a, 2a, 3a, 4a, 5a 5f, 6a 6f, 7a, 7b R ${ }^{1}=\mathrm{R}^{2}=\mathrm{CH}_{3} ; 1 \mathrm{~b}, 2 \mathrm{~b}, 3 \mathrm{~b}, 4 \mathrm{~b}, 5 \mathrm{~A} \sim 5 \mathrm{~F}, 6 \mathrm{~A} \sim 6 \mathrm{~F}, 7 \mathrm{~A}, 7 \mathrm{~B} \mathrm{R}^{1}+\mathrm{R}^{2}=\left(\mathrm{CH}_{2}\right)_{5}$

Scheme 2 Synthetic routes of the hydrazinyl tetronic acid derivatives and pyrazole-4-carboxylates

ported in our previous paper, and their spectra were consistent with the reported data. ${ }^{[17]}$ The target compounds $(\mathbf{5 a} \sim 5 \mathbf{5 f}, 5 \mathrm{~A} \sim \mathbf{5 F}, \mathbf{6 c} \sim \mathbf{6 f}, \mathbf{6 C} \sim \mathbf{6 F})$ were obtained by reflux 3 (route $A$ ) or $\mathbf{4}$ with different hydrazines in ethanol. The results showed that $\mathbf{3}$ or $\mathbf{4}$ could react smoothly with alkylhydrazine, benzylhydrazine, aromatic hydrazine, heterocyclichydrazine and benzoylhydrazine to afford target compounds. Compounds $\mathbf{6 a} \sim \mathbf{6 f}$ and $\mathbf{6 A} \sim \mathbf{6 F}$ were also prepared via the intermediates $\mathbf{2}$ and different hydrazines in ethanol at reflux temperature (route B). The $Z / E$ isomers were observed from their ${ }^{1} \mathrm{H}$ NMR spectra of compounds $6 \mathbf{6} \sim \mathbf{6 f}$ and $6 \mathbf{A} \sim 6 \mathbf{6 F}$, which are similar to that of their amino analogues $6 \mathrm{~g}$ and $\mathbf{6 G} \sim \mathbf{6 H}$ in the previous reports, and were further comfirmed by the ${ }^{13} \mathrm{C} \mathrm{NMR}$ spectra that showed characteristic carbon signals existing in pairs with similar peak heights. ${ }^{[17]}$

It was interesting that the reactions between the intermediates 3a and $\mathbf{3 b}$ with hydrazine hydrate or methylhydrazine yielded methyl 5-methyl-1H-pyrazole- 4-carboxylates $\mathbf{7 a}, \mathbf{7 b}, \mathbf{7 A}$ and $\mathbf{7 B}$, unexpectedly. The structure of the representative compound $\mathbf{7 a}$ was confirmed by X-ray crystallographic analysis, and depicted in Figure 3. However, the similar products were not obtained by the reaction of the intermediates $\mathbf{3 a}$ and $\mathbf{3 b}$ with other hydrazines in Scheme 2. A possible explanation for the phenomenon might be that the electron-withdrawing inductive effects and steric hindrance of $N$-substituent group such as phenyl, pyridyl and benzoyl weaken the nucleophilicity of the secondary amino group. In the other side, the ethyl esters were not obtained even the reactions were run in ethanol. But the ethyl ester 8 was afforded when we replaced methoxy intermediate 3a with ethoxy analog 3c (Figure 4a) in the reaction which was run both in ethanol and tetrahydrofuran (THF). It was suggested that the methyl of 7 or ethyl of $\mathbf{8}$ in the esters come from the methyl in 3a or ethyl of its ethyl analog 3c. Generally, this reaction was considered to go through the pathway A, in which the condensation as well as Michael addition-elimination casade reaction occurred to give a pyrazole with leaving methanol. Then methanol attacked the lactone to yield

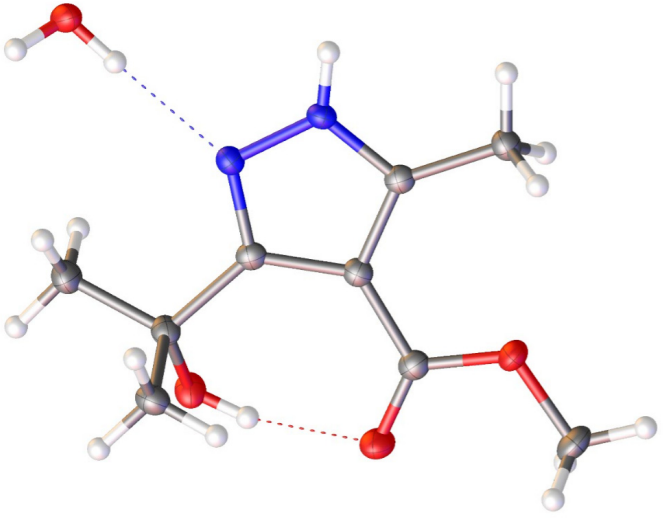

Figure 3 Crystal structure of compound 7a

methyl 5-methyl-1H-pyrazole-4-carboxylate (Figure 4b, path A). However, since ethanol was used as the solvent, ethanol also could attack carbonyl group of the lactone competitively to yield compound $\mathbf{8}$ in this pathway. The absence of compound $\mathbf{8}$ in our experiments did not support the A pathway. Our rational hypothesis for the mechanism is that the intermediate $\mathbf{3 a}$ reacted with hydrazine hydrate to form 3-methoxy-3,6,6-trimethyl-2,3,3a,6-tetrahydro-4Hfuro[3,4-c]pyrazol-4-one through the Michael addition and condensation reaction. Then methoxy group directly attacked the lactone carbonyl, and the intramolecular rearrangement was taken place to afford compound 7a (Figure $4 \mathrm{~b}$, path B). Then, the other hydrazines were used to react with intermediates $\mathbf{2 a}$ and $\mathbf{2} \mathbf{b}$ instead of hydrazine hydrate, compounds $\mathbf{6 a} \sim \mathbf{6 f}, \mathbf{6 A} \sim \mathbf{6 F}$ were obtained without pyrazole-4-carboxylates, this results indicated that both intermediates $\mathbf{2}$ and $\mathbf{3}$ could be utilized to synthesize compound $\mathbf{6}$ with no significant yield differences. Compounds $\mathbf{2 a}$ and $\mathbf{2 b}$ were dicarbonyl compounds, two regioisomers were expected to be produced when they reacted with substituted hydrazines. ${ }^{[28]}$ However, the isomer 5 was not obtained but compound $\mathbf{6}$ was the sole final product.

All the novel compounds including compounds $\mathbf{5 g} \sim \mathbf{5} \mathbf{k}$, $5 \mathrm{G} \sim 5 \mathrm{~K}, \mathbf{6 g} \sim \mathbf{6 h}$ and $\mathbf{6 G} \sim \mathbf{6 H}$ synthesized in the previous 
a

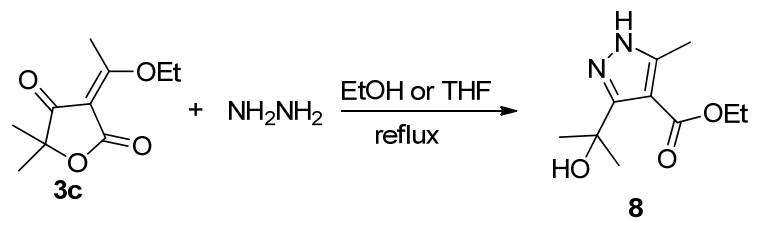

b proposed reaction mechanism

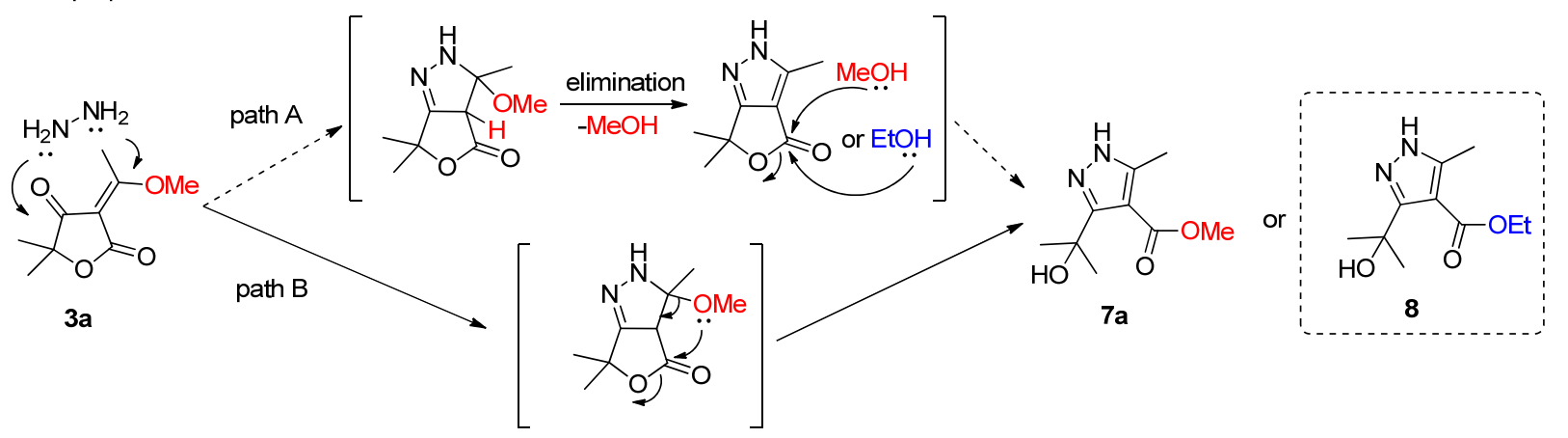

Figure 4 Proposed reaction mechanism

report were screened for the in vivo activities against Pseudoperonospora cubensis, Erysiphe graminis, Puccinia polysora and Colletotrichum llagenarium, and the in vitro activities against Pyricularia grisea and Botrytis cinerea. Fluormorphy, pyraclostrobin, and prochloraz were used as the positive controls. The results were shown in Table 1 .

The data in Table 1 clearly indicated that some of the amino derivatives exhibited significantly higher fungicidal activities than hydrazinyl ones, and much higher activities were observed for the C-4-substituted compounds compared with C-3-substituted derivatives for the in vivo activity. Especially, compounds 5G, 5H, 5i and 5I were observed $100 \%$ control efficacy against $C$. lagenarium at the concentration of $400 \mu \mathrm{g} / \mathrm{mL}$, which are comparable to the commercial control prochloraz. When the concentration decreased to 100,25 and $6.25 \mu \mathrm{g} / \mathrm{mL}$, compound $5 \mathbf{i}$ still displayed $100 \%, 60 \%$ and $20 \%$ control efficacy, respectively. When the substituents were alkyl amino (methylamino and dimethylamino), both 5G and 5H showed $98 \% \sim 100 \%$ control efficacy against E. graminis, $P$. polysora and $C$. lagenarium, which is comparable to the commercial control pyraclostrobin. Furthermore, the 5 -substituents were closely related with the fungicidal activity. The introduction of 5,5-spirocyclohexyl moiety significantly improved the fungicidal activity of the derivatives. The results may be rationalized due to unique properties of spiro compounds such as spiro-conjugated, spiro-superconjugation and anomeric effects. It has been reported that the anomeric effect could make the molecular more stable, in turn leading to the excellent biological activity. ${ }^{[29]}$ In the in vitro activity bioassy, the hydrazinyl derivatives possessed obvious differences. The derivatives 5c, 5d, 6c, 6d, 5C, 5D, 6C and 6D exhibited 50\% 80\% inhibition activities against $B$. cinerea, whereas compounds 5A, 5G, 6e and 6E showed $80 \%, 80 \%, 50 \%$ and $80 \%$ inhibition activities against $P$. grisea at $25 \mu \mathrm{g} / \mathrm{mL}$, respectively. The activities of compounds with phenylhyrazinyl and $p$-methoxyl phenylhyrazinyl groups were significantly improved for $B$. cinerea, which was consistent with the results reported in the literature. ${ }^{[19]}$ It was speculated that the arylhydrazine group was an electron-rich group, in which nitrogen can act as a hydrogen bond donor leading to interaction with various biological enzymes. ${ }^{[30]} 5,5$-Spirocycle compounds had much higher fungicidal activities than the 5,5-dimethyl compounds. These results clearly demonstrated that the spirocyclic moiety in tetronic acid scaffold had positive influence on the fungicidal activities, and the similar structure-activity relationships were observed. ${ }^{[13-15]}$ Compounds 5G and 5i exhibited excellent fungicidal activities against the tested phytopathagens. They could be the potential lead compounds for further optimization.

The insecticidal and acaricidal activities of the synthesized compounds against Plutella xylostella, Mythimna seprata, Myzus persicae and Tetranychus cinnabarinus were assessed and dipicted in Table 2. Some of the hydrazinyl derivatives possessed moderate to excellent insecticidal activities against the target insects except $M$. seprata. For example, compounds 6A, 69 and $\mathbf{6 H}$ showed $100 \%$ mortality against $M$. Persicae, compounds $\mathbf{5 b}, \mathbf{5 E}$ and 6F exhibted $100 \%$ mortality against $P$. xylostella, compound $\mathbf{6 b}$ indicated $100 \%$ mortality against $T$. cinnabarinus at the concentration of $600 \mu \mathrm{g} / \mathrm{mL}$. When the concentrations were decreased to 100 and $10 \mu \mathrm{g} / \mathrm{mL}$, the mortalities of compounds $\mathbf{5 b}$ and $\mathbf{5 E}$ against $P$. xylostella were $25 \%$ and $5.6 \%, 23.5$ and $20 \%$, the mortalities of compound $\mathbf{6 b}$ against $T$. cinnabarinus were $100 \%$ and $21.3 \%$, respectively. They could be used as valuable lead compounds for further modification in the future. These results confirmed that the hydrazinyl tetronic acid derivatives had much higher insecticidal and acaricidal activities compared with the amino-substituted derivatives. However, we found that the 5,5-dimethyl, 5,5-spirocycle substituents, and hydrazinyl regioisomers at C-3 and C-4 in these molecules did not show clear structure-activity 
Table 1 in vitro and in vivo fungicidal activities of title compounds against several phytopathagens
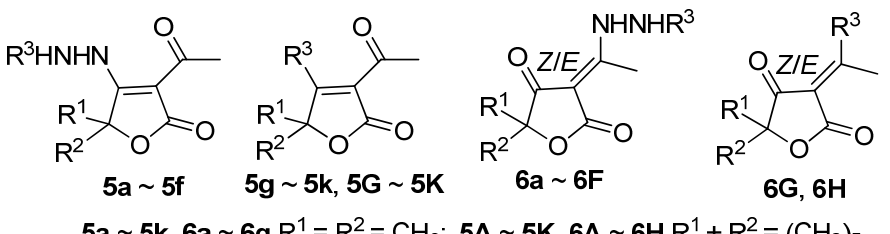

$5 \mathbf{a} \sim 5 \mathbf{k}, 6 \mathbf{a} \sim 6 \mathbf{g ~ R} \mathrm{R}^{1}=\mathrm{R}^{2}=\mathrm{CH}_{3} ; 5 \mathrm{~A} \sim 5 \mathrm{~K}, 6 \mathrm{~A} \sim 6 \mathrm{H} \mathrm{R}^{1}+\mathrm{R}^{2}=\left(\mathrm{CH}_{2}\right)_{5}$

\begin{tabular}{|c|c|c|c|c|c|c|c|}
\hline \multirow{2}{*}{ Compd. } & \multirow{2}{*}{$\mathrm{R}^{3}$} & \multicolumn{4}{|c|}{ Efficacy $/ \%$ in vivo $(400 \mu \mathrm{g} / \mathrm{mL})$} & \multicolumn{2}{|c|}{ Efficacy $/ \%$ in vitro $(25 \mu \mathrm{g} / \mathrm{mL})$} \\
\hline & & P. cubensis & E. graminis & P. polysora & C. lagenarium & P. grisea & B. cinerea \\
\hline $5 \mathbf{5 a}$ & $\mathrm{H}$ & 0 & 30 & 0 & 0 & 0 & 0 \\
\hline $\mathbf{5 A}$ & $\mathrm{H}$ & 0 & 0 & 0 & 0 & 80 & 50 \\
\hline $5 \mathbf{b}$ & $\mathrm{Me}$ & 0 & 0 & 0 & 0 & 0 & 0 \\
\hline 5B & $\mathrm{Me}$ & 0 & 0 & 0 & 0 & 0 & 0 \\
\hline $5 c$ & $\mathrm{C}_{6} \mathrm{H}_{5}$ & 0 & 0 & 0 & 0 & 0 & 80 \\
\hline $5 \mathrm{C}$ & $\mathrm{C}_{6} \mathrm{H}_{5}$ & 0 & 0 & 0 & 0 & 0 & 50 \\
\hline 5d & $\mathrm{MeOC}_{6} \mathrm{H}_{4}$ & 0 & 0 & 0 & 0 & 0 & 80 \\
\hline 5D & $\mathrm{MeOC}_{6} \mathrm{H}_{4}$ & 0 & 0 & 0 & 0 & 0 & 80 \\
\hline $5 e$ & 2-Py & 0 & 0 & 0 & 0 & 0 & 0 \\
\hline $\mathbf{5 E}$ & 2-Py & 0 & 0 & 0 & 0 & 0 & 0 \\
\hline $5 f$ & $\mathrm{C}_{6} \mathrm{H}_{5} \mathrm{CO}$ & 0 & 0 & 0 & 0 & 0 & 0 \\
\hline $5 F$ & $\mathrm{C}_{6} \mathrm{H}_{5} \mathrm{CO}$ & 0 & 0 & 0 & 0 & 0 & 0 \\
\hline $5 g$ & MeNH & 0 & 0 & 0 & 0 & 0 & 0 \\
\hline $5 G$ & $\mathrm{MeNH}$ & 30 & 100 & 100 & 100 & 80 & 0 \\
\hline $5 \mathbf{h}$ & $\mathrm{Me}_{2} \mathrm{~N}$ & 0 & 0 & 0 & 0 & 0 & 0 \\
\hline $\mathbf{5 H}$ & $\mathrm{Me}_{2} \mathrm{~N}$ & 0 & 98 & 100 & 100 & 0 & 0 \\
\hline $5 \mathbf{i}$ & HONH & 0 & 0 & 0 & $100,100^{a}, 60^{b}, 20^{c}$ & 0 & 0 \\
\hline $5 I$ & HONH & 0 & 0 & 0 & 100 & 0 & 0 \\
\hline $6 a$ & $\mathrm{H}$ & 0 & 0 & 0 & 0 & 0 & 0 \\
\hline $6 A$ & $\mathrm{H}$ & 0 & 0 & 0 & 0 & 0 & 0 \\
\hline $6 \mathbf{b}$ & $\mathrm{Me}$ & 0 & 0 & 0 & 0 & 0 & 0 \\
\hline $6 B$ & $\mathrm{Me}$ & 0 & 0 & 0 & 0 & 0 & 0 \\
\hline $6 c$ & $\mathrm{C}_{6} \mathrm{H}_{5}$ & 0 & 0 & 0 & 0 & 0 & 80 \\
\hline $6 \mathrm{C}$ & $\mathrm{C}_{6} \mathrm{H}_{5}$ & 0 & 0 & 0 & 0 & 0 & 80 \\
\hline $6 d$ & $4-\mathrm{MeOC}_{6} \mathrm{H}_{4}$ & 0 & 0 & 0 & 0 & 0 & 80 \\
\hline 6D & 4- $\mathrm{MeOC}_{6} \mathrm{H}_{4}$ & 0 & 0 & 0 & 0 & 0 & 80 \\
\hline $6 e$ & 2-Py & 0 & 40 & 0 & 0 & 50 & 0 \\
\hline $6 E$ & 2-Py & 0 & 0 & 0 & 0 & 80 & 0 \\
\hline $6 f$ & $\mathrm{C}_{6} \mathrm{H}_{5} \mathrm{CO}$ & 0 & 0 & 0 & 0 & 0 & 0 \\
\hline $6 F$ & $\mathrm{C}_{6} \mathrm{H}_{5} \mathrm{CO}$ & 0 & 0 & 0 & 0 & 0 & 0 \\
\hline $6 \mathrm{~g}$ & MeNH & 0 & 0 & 0 & 0 & 0 & 0 \\
\hline $6 G$ & $\mathrm{MeNH}$ & 40 & 0 & 0 & 0 & 0 & 0 \\
\hline \multicolumn{2}{|c|}{ Fluormorphy } & 100 & - & - & - & - & 0 \\
\hline \multicolumn{2}{|c|}{ Pyraclostrobin } & - & 100 & 100 & - & 100 & 100 \\
\hline \multicolumn{2}{|l|}{ Prochloraz } & - & - & - & 100 & - & - \\
\hline
\end{tabular}

${ }^{a}$ Activity at $100 \mu \mathrm{g} / \mathrm{mL} .{ }^{b}$ Activity at $25 \mu \mathrm{g} / \mathrm{mL} .{ }^{c}$ Activity at $6.25 \mu \mathrm{g} / \mathrm{mL}$. “—”: Not tested.

relationship for their insecticidal activities as they displayed for their fungicidal activities.

\section{Conclusion}

In summary, twenty four novel tetronic acid derivatives were synthesized through a Michael addition-elimination cascade reaction, four methyl 3-methyl-1H-pyrazole4-carboxylates were obtained unexpected, and a rational mechanism was proposed. Their structures were character- ized by HR-ESI-MS, ${ }^{1} \mathrm{H}$ NMR, ${ }^{13} \mathrm{C}$ NMR and X-ray crystallographic analysis. The bioassay results of the hydrazinyl compounds along with the amino analogues indicated that some of these compounds exhibited moderate to excellent fungicidal and insecticidal activities against phytopathagens and insects. For example, compounds 5G, 5H, 5I and 5i showed $100 \%$ in vivo inhibition against $C$. lagenarium, and compound 5G also exhibited $100 \%$ in vivo inhibition against E. graminis, P. polysora and C. lagenarium at 400 
Table 2 Insecticidal activities of title compounds against several insects.

\begin{tabular}{|c|c|c|c|c|c|}
\hline \multirow{2}{*}{ Compd. } & \multirow{2}{*}{$\mathrm{R}^{3}$} & \multicolumn{4}{|c|}{ Mortality (\%) at $600 \mu \mathrm{g} / \mathrm{mL}$} \\
\hline & & P. xylostella & M. separata & M. persicae & T. cinnabarinus \\
\hline $5 \mathbf{5 a}$ & $\mathrm{H}$ & 0 & 0 & 0 & 0 \\
\hline $5 \mathbf{A}$ & $\mathrm{H}$ & 0 & 0 & 80 & 37.5 \\
\hline $5 \mathbf{b}$ & $\mathrm{Me}$ & $100,25^{a}, 5.6^{b}$ & 0 & 0 & 0 \\
\hline 5B & $\mathrm{Me}$ & 0 & 0 & 0 & 0 \\
\hline $5 c$ & $\mathrm{Ph}$ & 0 & 0 & 0 & 0 \\
\hline $5 \mathrm{C}$ & $\mathrm{Ph}$ & 0 & 0 & 0 & 0 \\
\hline 5d & 4- $\mathrm{MeOC}_{6} \mathrm{H}_{4}$ & 0 & 0 & 0 & 50 \\
\hline 5D & $4-\mathrm{MeOC}_{6} \mathrm{H}_{4}$ & 0 & 0 & 0 & 0 \\
\hline $5 e$ & 2-Py & 0 & 0 & 0 & 0 \\
\hline $5 \mathbf{E}$ & 2-Py & $100,23.5^{a}, 20^{b}$ & 0 & 0 & 0 \\
\hline $5 f$ & $\mathrm{C}_{6} \mathrm{H}_{5} \mathrm{CO}$ & 0 & 0 & 0 & 0 \\
\hline $5 F$ & $\mathrm{C}_{6} \mathrm{H}_{5} \mathrm{CO}$ & 0 & 0 & 0 & 0 \\
\hline 5J & $\mathrm{HOCH}_{2} \mathrm{CH}_{2} \mathrm{NH}$ & 0 & 0 & 60 & 0 \\
\hline $5 \mathbf{k}$ & 2-PyNH & 0 & 0 & 0 & 37.5 \\
\hline $6 a$ & $\mathrm{H}$ & 0 & 0 & 37.5 & 0 \\
\hline $6 \mathrm{~A}$ & $\mathrm{H}$ & 0 & 0 & 100 & 0 \\
\hline $6 b$ & $\mathrm{Me}$ & 0 & 0 & 0 & $100,100^{a}, 21.3^{b}$ \\
\hline $6 B$ & $\mathrm{Me}$ & 0 & 0 & 0 & 0 \\
\hline $6 c$ & $\mathrm{Ph}$ & 0 & 0 & 0 & 0 \\
\hline $6 \mathrm{C}$ & $\mathrm{Ph}$ & 0 & 0 & 25.2 & 0 \\
\hline $6 d$ & $4-\mathrm{MeOC}_{6} \mathrm{H}_{4}$ & 0 & 0 & 0 & 0 \\
\hline 6D & $4-\mathrm{MeOC}_{6} \mathrm{H}_{4}$ & 0 & 0 & 0 & 0 \\
\hline $6 e$ & 2-Py & 0 & 0 & 0 & 0 \\
\hline $6 \mathrm{E}$ & 2-Py & 0 & 0 & 0 & 0 \\
\hline $6 f$ & $\mathrm{C}_{6} \mathrm{H}_{5} \mathrm{CO}$ & 0 & 0 & 0 & 0 \\
\hline $6 F$ & $\mathrm{C}_{6} \mathrm{H}_{5} \mathrm{CO}$ & $100,12.5^{a}, 11.1^{b}$ & 0 & 0 & 0 \\
\hline $6 \mathrm{~g}$ & $\mathrm{MeNH}$ & 0 & 0 & 100 & 0 \\
\hline $6 \mathrm{H}$ & $\mathrm{HOCH}_{2} \mathrm{CH}_{2} \mathrm{NH}$ & 0 & 0 & 100 & 0 \\
\hline \multicolumn{2}{|c|}{ Spirodiclofen } & - & - & - & $100^{b}$ \\
\hline \multicolumn{2}{|c|}{ Imidacloprid } & $100^{b}$ & $100^{b}$ & $100^{b}$ & $100^{b}$ \\
\hline
\end{tabular}

${ }^{a}$ Activity at $100 \mu \mathrm{g} / \mathrm{mL} .{ }^{b}$ Activity at $10 \mu \mathrm{g} / \mathrm{mL}$ "—”": Not tested.

$\mu \mathrm{g} / \mathrm{mL}$. Compounds $\mathbf{5 b}, \mathbf{5 E}$ and $\mathbf{6 F}$ showed $100 \%$ mortality against $P$. xylostella, compounds 6A, 6g and $\mathbf{6 H}$ had $100 \%$ mortality against $M$. persicae, and compound $\mathbf{6 b}$ had $100 \%$ mortality against $T$. cinnabarinus at $600 \mu \mathrm{g} / \mathrm{mL}$, respectively. The 5,5-spirocyclohexyl moiety significantly improved the fungicidal activities of the tetronic acid derivatives, and the introduction of the substituted hydrazinyl group to the tetronic acid skeleton led to higher insecticidal and acaricidal activities. 5G and 5i had the most potential to be further modified for searching new fungicide as the lead compounds.

\section{Experimental}

\subsection{General information}

All chemicals such as starting materials and reagents were purchased from commercial supplier (Energy Chemical) and used without further purification except as indicated. Organic solvents were concentrated under reduced pressure using a rotary evaporator or oil pump. All reactions were carried out using magnetic stirring. The silica gel chromatography was performed using 200 300 mesh flash silca gel (Qingdao Haiyang). Melting points (m.p.) were examined using a Yanagimoto apparatus without further corrected. ${ }^{1} \mathrm{H}$ NMR and ${ }^{13} \mathrm{C}$ NMR spectra were recorded on a Bruker DPX 300 spectrometer with $\mathrm{CDCl}_{3}$ or DMSO- $d_{6}$ as the solvents and tetramethylsilane (TMS) as the internal standard. HR-ESI-MS data were acquired on a Linear Trap Quadropole (LTQ) Orbitrap instrument equipped with electrospray ionization source (ESI). Crystal structure was analyzed at Thermo Fisher ESCALAB 250 X-ray diffractometry.

3.2 Synthesis of compounds $5 a \sim 6 \mathrm{~F}, 5 \mathrm{~g} \sim 5 \mathrm{i}, 5 \mathrm{G} \sim$ 5I, 6g, 6G, 7a, 7b, 7A and 7B

Compounds $5 \mathrm{~g} \sim \mathbf{5 i}, \mathbf{5 G} \sim \mathbf{5 I}, \mathbf{6 g}$ and $\mathbf{6 G}$ were synthesized following the procedures and the spectral data were identical with that reported in literature. ${ }^{[17]}$ The general synthetic procedures for compounds $\mathbf{5 a} \sim \mathbf{6 F}, \mathbf{7 a}, \mathbf{7 b}, \mathbf{7 A}$ and 7B are shown in Scheme 2. Took the synthesis of compound $\mathbf{6 c}$ as an example.

To a solution of compound $\mathbf{3 a}(184 \mathrm{mg}, 1.0 \mathrm{mmol}$, route A) or compound $\mathbf{2 a}(170 \mathrm{mg}, 1.0 \mathrm{mmol}$, route $\mathrm{B})$ in $20 \mathrm{~mL}$ of ethanol or THF, phenylhydrazine (108 mg, $1.0 \mathrm{mmol}, 1$ 
equiv.) was added. The reaction mixture was then heated to reflux in an oil bath. After $2 \sim 4 \mathrm{~h}$ of reaction, the mixture was cooled to room temperature and the solvent was removed under reduced pressure. Then the residue was purified by silica gel chromatograph and eluented with petroleum/ethyl acetate $(V: V=5: 1$ to $2: 1)$ to afford a colorless crystal 6c $223 \mathrm{mg}$ (route A, yield 86\%), or $229 \mathrm{mg}$ (route B, yield 89\%). The other compounds were prepared in a similar approaches.

3-Acetyl-4-hydrazinyl-5,5-dimethylbutenolide

(5a): Yield 79\%. Colorless crystal, m.p. $175 \sim 176{ }^{\circ} \mathrm{C} ;{ }^{1} \mathrm{H}$ NMR $\left(300 \mathrm{MHz}, \mathrm{CDCl}_{3}\right) \delta: 10.61$ (brs, 1H, NH), 4.08 (s, 2H, $\left.\mathrm{NH}_{2}\right), 2.50\left(\mathrm{~s}, 3 \mathrm{H}, \mathrm{CH}_{3}\right), 1.78\left(\mathrm{~s}, 6 \mathrm{H}, 2 \times \mathrm{CH}_{3}\right) ;{ }^{13} \mathrm{C} \mathrm{NMR}$ (75 MHz) $\delta$ : 196.72, 179.84, 168.61, 93.97, 79.72, 27.14, 24.16. HR-ESI-MS calcd for $\mathrm{C}_{8} \mathrm{H}_{13} \mathrm{~N}_{2} \mathrm{O}_{3}[\mathrm{M}+\mathrm{H}]{ }^{+}$ 185.0921, found 185.0924.

3-Acetyl-4-hydrazinyl-1-oxaspiro[4.5]dec-3-en-2-one (5A): Yield 60\%. Colorless crystal, m.p. 54 55 ${ }^{\circ} \mathrm{C} ;{ }^{1} \mathrm{H}$ NMR (300 MHz, $\mathrm{CDCl}_{3}$ ) $\delta: 10.57$ (brs, 1H, NH), 5.09 (s, $\left.2 \mathrm{H}, \mathrm{NH}_{2}\right), 2.26\left(\mathrm{~s}, 3 \mathrm{H}, \mathrm{CH}_{3}\right), 1.57 \sim 1.19\left(\mathrm{~m}, 10 \mathrm{H}, 5 \times \mathrm{CH}_{2}\right)$; ${ }^{13} \mathrm{C}$ NMR (75 MHz) $\delta$ : 194.34, 177.57, 168.94, 92.41, $81.22,31.67,27.19,24.21,21.42$. HR-ESI-MS calcd for $\mathrm{C}_{11} \mathrm{H}_{17} \mathrm{~N}_{2} \mathrm{O}_{3}[\mathrm{M}+\mathrm{H}]^{+}$225.1234, found 225.1231.

3-Acetyl-4-methylhydrazinyl-5,5-dimethylbutenolide (5b): Yield 81\%. Colorless crystal, m.p. $79 \sim 81{ }^{\circ} \mathrm{C} ;{ }^{1} \mathrm{H}$ NMR (300 MHz, $\mathrm{CDCl}_{3}$ ) $\delta: 10.35$ (brs, 1H, NH), 4.12 (q, $J=6.0 \mathrm{~Hz}, 1 \mathrm{H}, \mathrm{NH}), 2.72\left(\mathrm{~d}, J=6.0 \mathrm{~Hz}, 3 \mathrm{H}, \mathrm{NCH}_{3}\right), 2.44$ $\left(\mathrm{s}, 3 \mathrm{H}, \mathrm{CH}_{3}\right), 1.67\left(\mathrm{~s}, 6 \mathrm{H}, 2 \times \mathrm{CH}_{3}\right) ;{ }^{13} \mathrm{C} \mathrm{NMR}(75 \mathrm{MHz}) \delta$ : 197.00, 179.31, 169.20, 93.93, 80.29, 40.06, 27.70, 24.76. HR-ESI-MS calcd for $\mathrm{C}_{9} \mathrm{H}_{15} \mathrm{~N}_{2} \mathrm{O}_{3}[\mathrm{M}+\mathrm{H}]^{+}$199.1077, found 199.1074 .

3-Acetyl-4-methylhydrazinyl-1-oxaspiro[4.5]dec-3-en2-one (5B): Yield 80\%. Colorless crystal, m.p. 80 $82{ }^{\circ} \mathrm{C}$; ${ }^{1} \mathrm{H} \mathrm{NMR}\left(300 \mathrm{MHz}, \mathrm{CDCl}_{3}\right) \delta: 10.39$ (brs, 1H, NH), 5.39 (q, $J=6.0 \mathrm{~Hz}, 1 \mathrm{H}, \mathrm{NH}), 2.53\left(\mathrm{~d}, J=6.0 \mathrm{~Hz}, 3 \mathrm{H}, \mathrm{NCH}_{3}\right), 2.28$ $\left(\mathrm{s}, 3 \mathrm{H}, \mathrm{CH}_{3}\right), 1.67 \sim 1.19\left(\mathrm{~m}, 10 \mathrm{H}, 5 \mathrm{xCH}_{2}\right) ;{ }^{13} \mathrm{C} \mathrm{NMR}(75$ MHz) $\delta: 194.91,177.84,169.04,92.98,81.78,32.48,27.76$, 24.53, 21.78. HR-ESI-MS calcd for $\mathrm{C}_{12} \mathrm{H}_{19} \mathrm{~N}_{2} \mathrm{O}_{3}[\mathrm{M}+\mathrm{H}]^{+}$ 239.1390, found 239.1391.

3-Acetyl-4-phenylhydrazinyl-5,5-dimethylbutenolide (5c): Yield 61\%. Colorless crystal, m.p. $181 \sim 183{ }^{\circ} \mathrm{C} ;{ }^{1} \mathrm{H}$ NMR (300 MHz, $\left.\mathrm{CDCl}_{3}\right) \delta: 10.67$ (brs, 1H, NH), 7.36 $7.30(\mathrm{~m}, 2 \mathrm{H}, \mathrm{ArH}), 7.08 \sim 7.02(\mathrm{~m}, 1 \mathrm{H}, \mathrm{ArH}), 6.88 \sim 6.85$ $(\mathrm{m}, 2 \mathrm{H}, \mathrm{ArH}), 6.48(\mathrm{~s}, 1 \mathrm{H}, \mathrm{NH}), 2.52\left(\mathrm{~s}, 3 \mathrm{H}, \mathrm{CH}_{3}\right), 1.67(\mathrm{~s}$, $\left.6 \mathrm{H}, 2 \times \mathrm{CH}_{3}\right) ;{ }^{13} \mathrm{C} \mathrm{NMR}(75 \mathrm{MHz}) \delta: 197.02,180.34$, $168.77,146.09,129.25,122.36,113.43,94.79,80.13$, 27.57, 24.55. HR-ESI-MS calcd for $\mathrm{C}_{14} \mathrm{H}_{17} \mathrm{~N}_{2} \mathrm{O}_{3}[\mathrm{M}+\mathrm{H}]^{+}$ 261.1234, found 261.1238.

3-Acetyl-4-phenylhydrazinyl-1-oxaspiro[4.5]dec-3-en2-one (5C): Yield 81\%. Colorless crystal, m.p. 185 $186{ }^{\circ} \mathrm{C} ;{ }^{1} \mathrm{H}$ NMR $\left(300 \mathrm{MHz}, \mathrm{CDCl}_{3}\right) \delta: 10.77$ (brs, $1 \mathrm{H}$, $\mathrm{NH}), 7.37 \sim 7.31(\mathrm{~m}, 2 \mathrm{H}, \mathrm{ArH}), 7.09 \sim 7.04(\mathrm{~m}, 1 \mathrm{H}, \mathrm{ArH})$, $6.89 \sim 6.85(\mathrm{~m}, 2 \mathrm{H}, \mathrm{ArH}), 6.29(\mathrm{~s}, 1 \mathrm{H}, \mathrm{NH}), 2.54(\mathrm{~s}, 3 \mathrm{H}$, $\left.\mathrm{CH}_{3}\right), 2.22 \sim 1.25\left(\mathrm{~m}, 10 \mathrm{H}, 5 \times \mathrm{CH}_{2}\right) ;{ }^{13} \mathrm{C} \mathrm{NMR}(75 \mathrm{MHz})$ $\delta: 197.31,180.04,168.86,146.07,129.31,122.48,113.58$, $95.22,82.04,32.53,27.63,23.95,21.15$. HR-ESI-MS calcd for $\mathrm{C}_{17} \mathrm{H}_{21} \mathrm{~N}_{2} \mathrm{O}_{3}[\mathrm{M}+\mathrm{H}]^{+}$301.1541, found 301.1538.
3-Acetyl-4-(4-methoxyphenyl)hydrazinyl-5,5-dimethylbutenolide (5d): Yield 85\%. Colorless crystal, m.p. 134 $136{ }^{\circ} \mathrm{C} ;{ }^{1} \mathrm{H}$ NMR $\left(300 \mathrm{MHz}, \mathrm{CDCl}_{3}\right) \delta: 10.62(\mathrm{~s}, 1 \mathrm{H}, \mathrm{NH})$, $8.14(\mathrm{~s}, 1 \mathrm{H}, \mathrm{NH}), 6.84$ (d, J=9.0 Hz, 2H, ArH), 6.72 (d, $J=9.0 \mathrm{~Hz}, 2 \mathrm{H}, \mathrm{ArH}), 3.67$ (s, 3H, $\left.\mathrm{OCH}_{3}\right), 2.33(\mathrm{~s}, 3 \mathrm{H}$, $\left.\mathrm{CH}_{3}\right), 1.54\left(\mathrm{~s}, 6 \mathrm{H}, 2 \times \mathrm{CH}_{3}\right) ;{ }^{13} \mathrm{C}$ NMR $(75 \mathrm{MHz}) \delta: 195.09$, $179.79,168.79,154.50,141.84,115.27,114.95,93.87$, $80.10,55.73,27.88,25.16$. HR-ESI-MS calcd for $\mathrm{C}_{15} \mathrm{H}_{19} \mathrm{~N}_{2} \mathrm{O}_{4}[\mathrm{M}+\mathrm{H}]^{+}$291.1339, found 291.1336.

3-Acetyl-4-(4-methoxyphenyl)hydrazinyl-1-oxaspiro[4.5]dec-3-en-2-one (5D): Yield 61\%. Colorless crystal, m.p. $181 \sim 184{ }^{\circ} \mathrm{C} ;{ }^{1} \mathrm{H} \mathrm{NMR}\left(300 \mathrm{MHz}, \mathrm{CDCl}_{3}\right) \delta: 10.67(\mathrm{~s}$, $1 \mathrm{H}, \mathrm{NH}), 8.14$ (s, 1H, NH), 6.86 (d, J=9.0 Hz, 2H, ArH), $6.73(\mathrm{~d}, J=9.0 \mathrm{~Hz}, 2 \mathrm{H}, \mathrm{ArH}), 3.69\left(\mathrm{~s}, 3 \mathrm{H}, \mathrm{OCH}_{3}\right), 2.34$ (s, $\left.3 \mathrm{H}, \mathrm{CH}_{3}\right), 2.21 \sim 1.46\left(\mathrm{~m}, 10 \mathrm{H}, 5 \times \mathrm{CH}_{2}\right) ;{ }^{13} \mathrm{C} \mathrm{NMR}(75$ MHz) $\delta: 195.03,179.11,168.58,154.26,141.57,115.11$, 114.68, 93.78, 81.41, 55.42, 32.30, 27.61, 24.13, 21.40. HR-ESI-MS calcd for $\mathrm{C}_{18} \mathrm{H}_{23} \mathrm{~N}_{2} \mathrm{O}_{4}[\mathrm{M}+\mathrm{H}]^{+} 331.1652$, found 331.1645 .

3-Acetyl-4-(2-pyridyl)hydrazinyl-5,5-dimethylbutenolide (5e): Yield 74\%. Colorless crystal, m.p. 83 85 ${ }^{\circ} \mathrm{C} ;{ }^{1} \mathrm{H}$ NMR (300 MHz, $\left.\mathrm{CDCl}_{3}\right) \delta: 10.07(\mathrm{~s}, 1 \mathrm{H}, \mathrm{NH}), 7.60 \sim 7.43$ $(\mathrm{m}, 2 \mathrm{H}, \mathrm{ArH}), 6.98 \sim 6.63(\mathrm{~m}, 2 \mathrm{H}, \mathrm{ArH}), 2.58\left(\mathrm{~s}, 3 \mathrm{H}, \mathrm{CH}_{3}\right)$, $1.15\left(\mathrm{~s}, 3 \mathrm{H}, \mathrm{CH}_{3}\right), 0.84\left(\mathrm{~s}, 3 \mathrm{H}, \mathrm{CH}_{3}\right) ;{ }^{13} \mathrm{C} \mathrm{NMR}(75 \mathrm{MHz}) \delta$ : $195.20,172.73,154.38,153.87,144.85,139.59,114.95$, $109.84,89.78,79.96,31.10,24.35,14.34$. HR-ESI-MS calcd for $\mathrm{C}_{13} \mathrm{H}_{16} \mathrm{~N}_{3} \mathrm{O}_{3}[\mathrm{M}+\mathrm{H}]^{+}$262.1186, found 262.1180 .

3-Acetyl-4-(2-pyridyl)hydrazinyl-1-oxaspiro[4.5]dec-3en-2-one (5E): Yield 52\%. Colorless crystal, m.p. 210 $212{ }^{\circ} \mathrm{C} ;{ }^{1} \mathrm{H}$ NMR $\left(300 \mathrm{MHz}, \mathrm{CDCl}_{3}\right) \delta: 9.61$ (s, $\left.1 \mathrm{H}, \mathrm{NH}\right)$, $8.19 \sim 7.64(\mathrm{~m}, 2 \mathrm{H}, \mathrm{ArH}), 6.92 \sim 6.79(\mathrm{~m}, 2 \mathrm{H}, \mathrm{ArH}), 2.55$ $\left(\mathrm{s}, 3 \mathrm{H}, \mathrm{CH}_{3}\right), 1.71 \sim 1.54\left(\mathrm{~m}, 10 \mathrm{H}, 5 \times \mathrm{CH}_{2}\right) ;{ }^{13} \mathrm{C} \mathrm{NMR}(75$ $\mathrm{MHz}) \delta$ : 198.65, 175.63, 167.18, 155.68, 147.56, 138.27, $116.65,108.59,87.57,81.55,32.18,24.40,21.41,13.27$. HR-ESI-MS calcd for $\mathrm{C}_{16} \mathrm{H}_{20} \mathrm{~N}_{3} \mathrm{O}_{3}[\mathrm{M}+\mathrm{H}]^{+}$302.1499, found 302.1496 .

3-Acetyl-4-benzoylhydrazinyl-5,5-dimethylbutenolide (5f): Yield 81\%. Colorless crystal, m.p. 107 108 ${ }^{\circ} \mathrm{C} ;{ }^{1} \mathrm{H}$ NMR (300 MHz, $\left.\mathrm{CDCl}_{3}\right) \delta$ : 10.99 (brs, $\left.1 \mathrm{H}, \mathrm{NH}\right), 10.87$ (brs, $1 \mathrm{H}, \mathrm{NH}), 7.88 \sim 7.50(\mathrm{~m}, 5 \mathrm{H}, \mathrm{ArH}), 2.36\left(\mathrm{~s}, 3 \mathrm{H}, \mathrm{CH}_{3}\right), 1.51$ $\left(\mathrm{s}, 6 \mathrm{H}, 2 \times \mathrm{CH}_{3}\right) ;{ }^{13} \mathrm{C} \mathrm{NMR}(75 \mathrm{MHz}) \delta: 194.99,179.02$, $168.58,132.89$, 132.06, 129.13, 128.07, 94.84, 79.83, 28.05, 25.08. HR-ESI-MS calcd for $\mathrm{C}_{15} \mathrm{H}_{17} \mathrm{~N}_{2} \mathrm{O}_{4}[\mathrm{M}+\mathrm{H}]^{+}$ 289.1183 , found 289.1186 .

3-Acetyl-4-benzoylhydrazinyl-1-oxaspiro[4.5]dec-3en-2-one (5F): Yield 72\%. Colorless crystal, m.p. 170 $172{ }^{\circ} \mathrm{C} ;{ }^{1} \mathrm{H}$ NMR $\left(300 \mathrm{MHz}, \mathrm{CDCl}_{3}\right) \delta: 11.01$ (s, 1H, NH), $10.89(\mathrm{~s}, 1 \mathrm{H}, \mathrm{NH}), 7.89 \sim 7.53(\mathrm{~m}, 5 \mathrm{H}, \mathrm{ArH}), 2.38(\mathrm{~s}, 3 \mathrm{H}$, $\left.\mathrm{CH}_{3}\right), 2.04 \sim 1.04\left(\mathrm{~m}, 10 \mathrm{H}, 5 \times \mathrm{CH}_{2}\right) ;{ }^{13} \mathrm{C} \mathrm{NMR}(75 \mathrm{MHz})$ $\delta: 194.92,178.28,168.35,132.58,131.82,128.87,127.74$, 94.88, 81.22, 32.48, 27.76, 24.39, 21.46. HR-ESI-MS calcd for $\mathrm{C}_{18} \mathrm{H}_{21} \mathrm{~N}_{2} \mathrm{O}_{4}[\mathrm{M}+\mathrm{H}]^{+}$329.1496, found 329.1486.

(E/Z)-3-(1-Hydrazinylethylidene)-5,5-dimethylfuran2,4-dione (6a): Yield 79\%. Colorless crystal, m.p. 162 $163{ }^{\circ} \mathrm{C} ;{ }^{1} \mathrm{H}$ NMR $\left(300 \mathrm{MHz}, \mathrm{CDCl}_{3}\right) \delta: 11.92,11.15$ (brs, $1 \mathrm{H}, \mathrm{NH}), 4.25$ (s, 2H, $\mathrm{NH}_{2}$ ), 2.69, 2.67 (s, 3H, $\left.\mathrm{CH}_{3}\right), 1.40$ (s, 
$\left.6 \mathrm{H}, 2 \times \mathrm{CH}_{3}\right) ;{ }^{13} \mathrm{C}$ NMR $(75 \mathrm{MHz}) \delta: 201.68,198.52$, $174.67,172.77,171.79,170.76,88.81,87.14,86.27,82.95$, 29.64, 23.77, 12.88. HR-ESI-MS calcd for $\mathrm{C}_{8} \mathrm{H}_{13} \mathrm{~N}_{2} \mathrm{O}_{3}$ $[\mathrm{M}+\mathrm{H}]^{+} 185.0921$, found 185.0926 .

(E/Z)-3-(1-Hydrazinylethylidene)-1-oxaspiro[4.5]decane-2,4-dione (6A): Yield 60\%. Colorless crystal, m.p. $54 \sim 55{ }^{\circ} \mathrm{C} ;{ }^{1} \mathrm{H}$ NMR $\left(300 \mathrm{MHz}, \mathrm{CDCl}_{3}\right) \delta$ : 11.62 (brs, $1 \mathrm{H}$, $\mathrm{NH}), 5.53\left(\mathrm{~s}, 2 \mathrm{H}, \mathrm{NH}_{2}\right), 2.46\left(\mathrm{~s}, 3 \mathrm{H}, \mathrm{CH}_{3}\right), 1.68 \sim 1.41(\mathrm{~m}$, $\left.10 \mathrm{H}, 5 \times \mathrm{CH}_{2}\right) ;{ }^{13} \mathrm{C}$ NMR $(75 \mathrm{MHz}) \delta: 198.16,171.92$, $165.94,86.11,83.76,32.19,24.41,21.44,12.57$. HR-ESIMS calcd for $\mathrm{C}_{11} \mathrm{H}_{17} \mathrm{~N}_{2} \mathrm{O}_{3}[\mathrm{M}+\mathrm{H}]^{+}$225.1234, found 225.1230 .

(E/Z)-3-(1-Methylhydrazinylethylidene)-5,5-dimethylfuran-2,4-dione (6b): Yield 71\%. Yellow liquid; ${ }^{1} \mathrm{H}$ NMR $\left(300 \mathrm{MHz}, \mathrm{CDCl}_{3}\right) \delta: 11.70,10.92$ (brs, 1H, NH), 4.21 (brs, $1 \mathrm{H}, \mathrm{NH}), 2.74$ (d, $\left.J=5.4 \mathrm{~Hz}, 3 \mathrm{H}, \mathrm{NCH}_{3}\right), 2.64,2.62$ (s, 3H, $\left.\mathrm{CH}_{3}\right), 1.38\left(\mathrm{~s}, 6 \mathrm{H}, 2 \times \mathrm{CH}_{3}\right) ;{ }^{13} \mathrm{C} \mathrm{NMR}(75 \mathrm{MHz}) \delta: 201.50$, $197.97,174.13,171.84,171.10,170.30,89.82,88.41$, $85.10,82.42,29.43,23.22,13.32$. HR-ESI-MS calcd for $\mathrm{C}_{9} \mathrm{H}_{15} \mathrm{~N}_{2} \mathrm{O}_{3}[\mathrm{M}+\mathrm{H}]^{+}$199.1077, found 199.1073.

(E/Z)-3-(1-Methylhydrazinylethylidene)-1-oxaspiro[4.5]decane-2,4-dione (6B): Yield 72\%. Colorless crystal, m.p. $101 \sim 103{ }^{\circ} \mathrm{C} ;{ }^{1} \mathrm{H}$ NMR $\left(300 \mathrm{MHz}, \mathrm{CDCl}_{3}\right) \delta$ : 11.74 , 10.91 (brs, $1 \mathrm{H}, \mathrm{NH}), 4.22$ (brs, $1 \mathrm{H}, \mathrm{NH}), 2.75$ (d, $J=5.4 \mathrm{~Hz}$, $\left.3 \mathrm{H}, \mathrm{NCH}_{3}\right), 2.63,2.62\left(\mathrm{~s}, 3 \mathrm{H}, \mathrm{CH}_{3}\right), 1.72 \sim 1.23(\mathrm{~m}, 10 \mathrm{H}$, $\left.5 \times \mathrm{CH}_{2}\right) ;{ }^{13} \mathrm{C}$ NMR $(75 \mathrm{MHz}) \delta: 201.18,198.23,174.43$, $171.79,171.14,170.82,88.81,87.64,87.36,84.36,38.68$, $38.63,31.99,31.81,24.32,24.29,21.14,21.09,12.92$, 12.69. HR-ESI-MS calcd for $\mathrm{C}_{12} \mathrm{H}_{19} \mathrm{~N}_{2} \mathrm{O}_{3}[\mathrm{M}+\mathrm{H}]{ }^{+}$ 239.1390, found 239.1394.

(E/Z)-3-(1-Phenylhydrazinylethylidene)-5,5-dimethylfuran-2,4-dione (6c): Yield $86 \%$ (route A) or $89 \%$ (route B). Colorless crystal, m.p. $126 \sim 127{ }^{\circ} \mathrm{C} ;{ }^{1} \mathrm{H}$ NMR $(300 \mathrm{MHz}$, $\left.\mathrm{CDCl}_{3}\right) \delta: 11.91,11.13(\mathrm{~s}, 1 \mathrm{H}, \mathrm{NH}), 7.33 \sim 6.79(\mathrm{~m}, 5 \mathrm{H}$, $\mathrm{ArH}), 6.43$ (s, 1H, NH), 2.69, $2.68\left(\mathrm{~s}, 3 \mathrm{H}, \mathrm{CH}_{3}\right), 1.44$ (s, 6H, $\left.2 \times \mathrm{CH}_{3}\right) ;{ }^{13} \mathrm{C}$ NMR $(75 \mathrm{MHz}) \delta: 202.03,198.92,174.55$, $174.10,173.84,170.56,145.47,129.63,122.46,113.46$, 89.67, 88.26, 86.74, 83.49, 23.79, 13.20. HR-ESI-MS calcd for $\mathrm{C}_{14} \mathrm{H}_{17} \mathrm{~N}_{2} \mathrm{O}_{3}[\mathrm{M}+\mathrm{H}]^{+}$261.1234, found 261.1239.

(E/Z)-3-(1-Phenylhydrazinylethylidene)-1-oxaspiro[4.5]decane-2,4-dione (6C): Yield 72\% (route A) or 70\% (route B). Colorless crystal, m.p. 162 163 ${ }^{\circ} \mathrm{C} ;{ }^{1} \mathrm{H}$ NMR $\left(300 \mathrm{MHz}, \mathrm{CDCl}_{3}\right) \delta: 11.95,11.14$ (brs, $\left.1 \mathrm{H}, \mathrm{NH}\right), 7.32 \sim$ 6.78 (m, 5H, ArH), 6.42 (brs, 1H, NH), 2.68, 2.67 (s, 3H, $\left.\mathrm{CH}_{3}\right), 1.79 \sim 1.60\left(\mathrm{~m}, 10 \mathrm{H}, 5 \times \mathrm{CH}_{2}\right) ;{ }^{13} \mathrm{C} \mathrm{NMR}(75 \mathrm{MHz})$ $\delta: 201.87,198.74,174.50,174.43,173.85,170.88,90.27$, $88.87,88.61,85.24,32.32,32.17,24.59,21.43,21.37$, 13.32, 13.18. HR-ESI-MS calcd for $\mathrm{C}_{17} \mathrm{H}_{21} \mathrm{~N}_{2} \mathrm{O}_{3}[\mathrm{M}+\mathrm{H}]^{+}$, 301.1541 , found 301.1538 .

(E/Z)-3-(1-(4-Methoxyphenyl)hydrazinylethylidene)5,5-dimethylfuran-2,4-dione (6d): Yield 88\% (route A) or $85 \%$ (route B). Colorless crystal, m.p. $134 \sim 136{ }^{\circ} \mathrm{C} ;{ }^{1} \mathrm{H}$ NMR (300 MHz, $\left.\mathrm{CDCl}_{3}\right) \delta: 11.89,11.11(\mathrm{~s}, 1 \mathrm{H}, \mathrm{NH})$, $6.88 \sim 6.74(\mathrm{~m}, 4 \mathrm{H}, \mathrm{ArH}), 6.08(\mathrm{~s}, 1 \mathrm{H}, \mathrm{NH}), 3.77(\mathrm{~s}, 3 \mathrm{H}$, $\left.\mathrm{OCH}_{3}\right), 2.70,2.69\left(\mathrm{~s}, 3 \mathrm{H}, \mathrm{CH}_{3}\right), 1.43\left(\mathrm{~s}, 6 \mathrm{H}, 2 \times \mathrm{CH}_{3}\right) ;{ }^{13} \mathrm{C}$ NMR (75 MHz) $\delta: 201.99,198.88,174.03,173.92,173.30$, $170.48,138.67,115.54,115.02,89.59,88.10,86.58,83.35$,
55.62, 23.77, 13.24. HR-ESI-MS calcd for $\mathrm{C}_{15} \mathrm{H}_{19} \mathrm{~N}_{2} \mathrm{O}_{4}$ $[\mathrm{M}+\mathrm{H}]^{+}$291.1339, found 291.1337.

(E/Z)-3-(1-(4-Methoxyphenyl)hydrazinylethylidene)-1oxaspiro[4.5]decane-2,4-dione (6D): Yield 60\% (route A) or $59 \%$ (route B). Colorless crystal, m. p. $87 \sim 89{ }^{\circ} \mathrm{C} ;{ }^{1} \mathrm{H}$ NMR (300 MHz, $\left.\mathrm{CDCl}_{3}\right) \delta: 11.83,11.04$ (brs, $\left.1 \mathrm{H}, \mathrm{NH}\right)$, 8.18 (brs, $1 \mathrm{H}, \mathrm{NH}), 6.90 \sim 6.77(\mathrm{~m}, 4 \mathrm{H}, \mathrm{ArH}), 3.69$ (s, 3H, $\left.\mathrm{OCH}_{3}\right), 2.56\left(\mathrm{~s}, 3 \mathrm{H}, \mathrm{CH}_{3}\right), 1.67 \sim 1.50\left(\mathrm{~m}, 10 \mathrm{H}, 5 \times \mathrm{CH}_{2}\right)$; ${ }^{13} \mathrm{C}$ NMR (75 MHz) $\delta: 208.14,200.26,197.25,191.40$, $172.25,154.26,140.01,115.11,114.87,102.69$, 99.65, $88.62, \quad 83.42, \quad 55.43, \quad 32.12, \quad 24.38, \quad 21.37, \quad 13.10$. HR-ESI-MS calcd for $\mathrm{C}_{18} \mathrm{H}_{23} \mathrm{~N}_{2} \mathrm{O}_{4}[\mathrm{M}+\mathrm{H}]^{+}$331.1652, found 331.1648 .

(E/Z)-3-(1-(2-Pyridyl)hydrazinylethylidene)-5,5-dimethylfuran-2,4-dione (6e): Yield $80 \%$ (route A) or $74 \%$ (route B). Colorless crystal, m.p. 83 85 ${ }^{\circ} \mathrm{C} ;{ }^{1} \mathrm{H}$ NMR $(300 \mathrm{MHz}$, $\left.\mathrm{CDCl}_{3}\right) \delta: 15.92,12.15,10.81,9.06(\mathrm{~s}, 2 \mathrm{H}, \mathrm{NH}), 8.16 \sim 8.14$ $(\mathrm{m}, 1 \mathrm{H}, \mathrm{ArH}), 7.73 \sim 7.62(\mathrm{~m}, 1 \mathrm{H}, \mathrm{ArH}), 6.89 \sim 6.85(\mathrm{~m}$, $1 \mathrm{H}, \mathrm{ArH}), 6.74 \sim 6.70(\mathrm{~m}, 1 \mathrm{H}, \mathrm{ArH}), 2.32,2.31(\mathrm{~s}, 3 \mathrm{H}$, $\left.\mathrm{CH}_{3}\right), 1.49,1.41\left(\mathrm{~s}, 6 \mathrm{H}, 2 \times \mathrm{CH}_{3}\right) ;{ }^{13} \mathrm{C} \mathrm{NMR}(75 \mathrm{MHz}) \delta$ : 206.80, 194.91, 191.31, 179.55, 171.56, 168.78, 159.16, $148.11,141.90,138.31,135.82,116.95,112.76,111.12$, 108.68, 94.14, 92.15, 79.99, 79.45, 31.09, 27.84, 27.02, 25.04. HR-ESI-MS calcd for $\mathrm{C}_{13} \mathrm{H}_{16} \mathrm{~N}_{3} \mathrm{O}_{3}[\mathrm{M}+\mathrm{H}]{ }^{+}$ 262.1186, found 262.1180 .

(E/Z)-3-(1-(2-Pyridyl)hydrazinylethylidene)-1-oxaspiro[4.5]decane-2,4-dione (6E): Yield 73\% (route A) or $67 \%$ (route B). Colorless crystal, m.p. 74 $75{ }^{\circ} \mathrm{C} ;{ }^{1} \mathrm{H}$ NMR $(300$ $\left.\mathrm{MHz}, \mathrm{CDCl}_{3}\right) \delta: 15.90,12.20,10.84,9.05(\mathrm{~s}, 2 \mathrm{H}, \mathrm{NH})$, $8.18 \sim 8.15(\mathrm{~m}, 1 \mathrm{H}, \mathrm{ArH}), 7.79 \sim 7.64(\mathrm{~m}, 1 \mathrm{H}, \mathrm{ArH}), 6.90 \sim$ $6.86(\mathrm{~m}, 1 \mathrm{H}, \mathrm{ArH}), 6.76 \sim 6.61(\mathrm{~m}, 1 \mathrm{H}, \mathrm{ArH}), 2.35,2.31(\mathrm{~s}$, $\left.3 \mathrm{H}, \mathrm{CH}_{3}\right), 2.10 \sim 1.49\left(\mathrm{~m}, 10 \mathrm{H}, 5 \times \mathrm{CH}_{2}\right) ;{ }^{13} \mathrm{C} \mathrm{NMR}(75$ MHz) $\delta: 194.86,190.75,178.79,171.62,168.57,158.84$, $147.84,141.23,138.05,116.74,110.78,108.53,94.09$, $91.91,81.35,80.34,35.63,32.18,27.63,26.78,24.59$, 24.13, 21.93, 21.38. HR-ESI-MS calcd for $\mathrm{C}_{16} \mathrm{H}_{20} \mathrm{~N}_{3} \mathrm{O}_{3}$ $[\mathrm{M}+\mathrm{H}]^{+}$302.1499, found 302.1495.

(E/Z)-3-(1-Benzoylhydrazinylethylidene)-5,5-dimethylfuran-2,4-dione (6f): Yield $76 \%$ (route A) or $81 \%$ (route B). Colorless crystal, m.p. $107 \sim 108{ }^{\circ} \mathrm{C} ;{ }^{1} \mathrm{H}$ NMR $(300 \mathrm{MHz}$, $\left.\mathrm{CDCl}_{3}\right) \delta: 11.62$ (brs, $\left.1 \mathrm{H}, \mathrm{NH}\right), 7.96 \sim 7.42(\mathrm{~m}, 6 \mathrm{H}, \mathrm{ArH}+$ $\mathrm{NH}), 2.56,2.53\left(\mathrm{~s}, 3 \mathrm{H}, \mathrm{CH}_{3}\right), 1.32\left(\mathrm{~s}, 6 \mathrm{H}, 2 \times \mathrm{CH}_{3}\right) ;{ }^{13} \mathrm{C}$ NMR $(75 \mathrm{MHz}) \delta: 196.70,172.55,167.73,166.08,159.63$, $134.35,133.58,131.61,129.13,128.82,128.61,127.99$, 99.97, 89.87, 80.49, 79.60, 24.28, 13.62. HR-ESI-MS calcd for $\mathrm{C}_{15} \mathrm{H}_{17} \mathrm{~N}_{2} \mathrm{O}_{4}[\mathrm{M}+\mathrm{H}]^{+}$289.1183, found 289.1175.

(E/Z)-3-(1-Benzoylhydrazinylethylidene)-1-oxaspiro[4.5]decane-2,4-dione (6F): Yield $60 \%$ (route A) or $64 \%$ (route B). Colorless crystal, m.p. $188 \sim 190{ }^{\circ} \mathrm{C} ;{ }^{1} \mathrm{H}$ NMR $\left(300 \mathrm{MHz}, \mathrm{CDCl}_{3}\right) \delta: 12.18,11.38,10.76,9.39$ (brs, 2H, $\mathrm{NH}), 7.93 \sim 7.48(\mathrm{~m}, 5 \mathrm{H}, \mathrm{ArH}), 2.54\left(\mathrm{~s}, 3 \mathrm{H}, \mathrm{CH}_{3}\right), 1.73 \sim$ $1.22\left(\mathrm{~m}, 10 \mathrm{H}, 5 \times \mathrm{CH}_{2}\right) ;{ }^{13} \mathrm{C} \mathrm{NMR}(75 \mathrm{MHz}) \delta: 200.08$, $197.43,172.58,170.38,169.66,165.55,132.66,131.45$, $128.76,128.65,127.86,127.52,89.08,87.78,83.70,80.75$, $33.98,32.13,27.76,24.36,21.86,21.35,13.17$. HR-ESIMS calcd for $\mathrm{C}_{18} \mathrm{H}_{21} \mathrm{~N}_{2} \mathrm{O}_{4}[\mathrm{M}+\mathrm{H}]^{+}$329.1496, found 329.1489 . 
Methyl 3-(2-hydroxypropan-2-yl)-5-methyl-1 $H$-pyrazole-4-carboxylate (7a): Yield 62\%. Colorless crystal, m.p. 201 202 ${ }^{\circ} \mathrm{C}$; ${ }^{1} \mathrm{H}$ NMR $\left(300 \mathrm{MHz}, \mathrm{CDCl}_{3}\right) \delta: 3.90(\mathrm{~s}, 3 \mathrm{H}$, $\left.\mathrm{OCH}_{3}\right), 2.46\left(\mathrm{~s}, 3 \mathrm{H}, \mathrm{CH}_{3}\right), 1.62\left(\mathrm{~s}, 6 \mathrm{H}, 2 \times \mathrm{CH}_{3}\right) ;{ }^{13} \mathrm{C} \mathrm{NMR}$ (75 MHz) $\delta: 166.50,159.85,147.27,106.87,69.10,51.46$, 28.40, 12.79. HR-ESI-MS calcd for $\mathrm{C}_{9} \mathrm{H}_{15} \mathrm{~N}_{2} \mathrm{O}_{3}[\mathrm{M}+\mathrm{H}]^{+}$ 199.1070, found 199.1077.

Methyl 3-(1-hydroxycyclohexyl)-5-methyl-1H-pyrazole4-carboxylate (7A): Yield 62\%. Colorless crystal, m.p. 213 $214{ }^{\circ} \mathrm{C} ;{ }^{1} \mathrm{H}$ NMR $\left(300 \mathrm{MHz}, \mathrm{CDCl}_{3}\right) \delta$ : 5.74 (brs, $\mathrm{H}$, $\mathrm{OH}), 3.90\left(\mathrm{~s}, 3 \mathrm{H}, \mathrm{OCH}_{3}\right), 2.48\left(\mathrm{~s}, 3 \mathrm{H}, \mathrm{CH}_{3}\right), 1.62(\mathrm{~s}, 10 \mathrm{H}$, $\left.5 \times \mathrm{CH}_{2}\right) ;{ }^{13} \mathrm{C}$ NMR $(75 \mathrm{MHz}) \delta: 166.55,160.12,147.57$, $107.05,69.76,51.40,29.33$, 13.00. HR-ESI-MS calcd for $\mathrm{C}_{13} \mathrm{H}_{21} \mathrm{~N}_{2} \mathrm{O}_{3}[\mathrm{M}+\mathrm{H}]^{+}$253.1381, found 238.1317.

Methyl 3-(2-hydroxypropan-2-yl)-1,5-dimethyl-1 $H$ pyrazole-4-carboxylate (7b): Yield 84\%. Colorless liquid; ${ }^{1} \mathrm{H}$ NMR (300 MHz, $\left.\mathrm{CDCl}_{3}\right) \delta: 6.74$ (brs, $\left.1 \mathrm{H}, \mathrm{OH}\right), 3.93$ (s, $\left.3 \mathrm{H}, \mathrm{OCH}_{3}\right), 3.85\left(\mathrm{~s}, 3 \mathrm{H}, \mathrm{NCH}_{3}\right), 2.34\left(\mathrm{~s}, 3 \mathrm{H}, \mathrm{CH}_{3}\right), 1.66(\mathrm{~s}$, $\left.6 \mathrm{H}, 2 \times \mathrm{CH}_{3}\right) ;{ }^{13} \mathrm{C} \mathrm{NMR}(75 \mathrm{MHz}) \delta: 167.64,154.48$, $150.32,109.25,69.56,51.84,40.80,29.70,14.95$. HR-ESIMS calcd for $\mathrm{C}_{10} \mathrm{H}_{17} \mathrm{~N}_{2} \mathrm{O}_{3}[\mathrm{M}+\mathrm{H}]^{+}$213.1239, found 213.1234 .

Methyl 3-(1-hydroxycyclohexyl)-1,5-dimethyl-1 $H$ pyrazole-4-carboxylate (7B): Yield 67\%. Colorless liquid; ${ }^{1} \mathrm{H}$ NMR $\left(300 \mathrm{MHz}, \mathrm{CDCl}_{3}\right.$ ) $\delta: 6.02$ (brs, 1H, OH), 4.00 (s, $\left.3 \mathrm{H}, \mathrm{OCH}_{3}\right), 3.86\left(\mathrm{~s}, 3 \mathrm{H}, \mathrm{NCH}_{3}\right), 2.34\left(\mathrm{~s}, 3 \mathrm{H}, \mathrm{CH}_{3}\right), 2.01 \sim$ $1.59\left(\mathrm{~m}, 10 \mathrm{H}, 5 \times \mathrm{CH}_{2}\right) ;{ }^{13} \mathrm{C} \mathrm{NMR}(75 \mathrm{MHz}) \delta: 167.29$, 154.29, 149.77, 109.21, 70.87, 51.44, 41.31, 35.97, 25.01, 20.96, 14.55. HR-ESI-MS calcd for $\mathrm{C}_{10} \mathrm{H}_{17} \mathrm{~N}_{2} \mathrm{O}_{3}[\mathrm{M}+\mathrm{H}]^{+}$ 253.1552, found 253.1546 .

Ethyl 3-(2-hydroxypropan-2-yl)-5-methyl-1 $H$-pyrazole-4-carboxylate (8): Yield 79\% (in ethanol) or 73\% (in THF). Colorless liquid; ${ }^{1} \mathrm{H}$ NMR (300 MHz, $\left.\mathrm{CDCl}_{3}\right) \delta: 6.85$ (brs, $1 \mathrm{H}, \mathrm{OH}), 4.32$ (q, $\left.J=7.1 \mathrm{~Hz}, 2 \mathrm{H}, \mathrm{OCH}_{2}\right), 3.93(\mathrm{~s}, 3 \mathrm{H}$, $\left.\mathrm{NCH}_{3}\right), 2.35\left(\mathrm{~s}, 3 \mathrm{H}, \mathrm{CH}_{3}\right), 1.65\left(\mathrm{~s}, 6 \mathrm{H}, 2 \times \mathrm{CH}_{3}\right), 1.37(\mathrm{t}$, $\left.J=7.1 \mathrm{~Hz}, 3 \mathrm{H}, \mathrm{CH}_{3}\right) ;{ }^{13} \mathrm{C} \mathrm{NMR}(75 \mathrm{MHz}) \delta: 166.90$, 154.09, 109.14, 69.20, 60.64, 40.44, 29.40, 14.76, 13.81. HR-ESI-MS calcd for $\mathrm{C}_{11} \mathrm{H}_{19} \mathrm{~N}_{2} \mathrm{O}_{3}[\mathrm{M}+\mathrm{H}]^{+}$227.1390, found 227.1399.

\subsection{X-Ray diffraction analysis of compound 7a}

The crystal of $7 \mathbf{a}$ was obtained from $n$-hexane/ethanol $(V: V=1: 20)$ solution. Its structure parameters were shown as following. crystal size: $0.34 \mathrm{~mm} \times 0.30$ $\mathrm{mm} \times 0.25 \mathrm{~mm}$, formula $\mathrm{C}_{9} \mathrm{H}_{16} \mathrm{~N}_{2} \mathrm{O}_{4}, M_{\mathrm{r}}=216.24$, monoclinic, $a=0.86880(2) \mathrm{nm}, b=0.84438(2) \mathrm{nm}, c=$ $1.54604(5) \mathrm{nm}, \beta=103.149(3)^{\circ}, V=1.10444(5) \mathrm{nm}^{3}, \rho=$ $1.300 \mathrm{mg} / \mathrm{mm}^{3}$, space group $P 2_{1} / n, Z=4, \mu($ Mo K $\alpha)=0.102$ $\mathrm{mm}^{-1}, F(000)=464, S=1.037$. Totally 4443 reflections were measured at $T=105.6 \mathrm{~K}, 2162$ unique reflections $\left(R_{\text {int }}=0.0236\right)$ were used for all calculations and structure refinement. The final $R_{1}$ was $0.0449, w R_{2}$ was 0.0877 (all data). The crystallographic data of $7 \mathbf{a}$ in this paper have been deposited with the Cambridge Crystallographic Data Centre with the accession number-CCDC 1470573. These data can be obtained free of charge from The Cambridge Crystallographic Data Centre via www.ccdc.cam.ac.uk/ data_request/cif.

\subsection{Biological activity evaluation of title compounds}

\subsubsection{Fungicidal activity bioassay}

Each of the synthetic compounds were dissolved into a solution of acetone and methanol $(V: V=1: 1)$ to produce a stock solution of concentration of $4000 \mathrm{mg} / \mathrm{L}$. All of evaluations were performed triplicates for each concentration and utilized the procedures in the references. ${ }^{[16,31,32]}$

The in vivo fungicidal activities of each synthetic compound against cucumber downy mildew (P. cubensis), white powder mildew (E. graminis), corn rust ( $P$. polysora) and anthracnose $(C$. lagenarium) were evaluateded as follows: whole plants were used in these experiments, series of test solutions were prepared by diluting the stock solution with aqueous solution containing $0.1 \%$ tween- 80 . The test solution was sprayed onto the host plants by means of a special plant sprayer. After $24 \mathrm{~h}$ of treatment, the plants were inoculated with the fungi and transferred into a greenhouse $\left(18 \sim 30{ }^{\circ} \mathrm{C}, \mathrm{RH}>50 \% \sim 60 \%\right)$. According to the specific infectious characteristics of the fungus, certain plants were then stored in a humidity-controlled chamber, and they were transferred to a greenhouse after the infection was obviously observed. Other non-infected plants were placed in the greenhouse directly. The activity of the compounds was ascertained by direct visual observation after $7 \mathrm{~d}$.

The in vitro biological activities against rice blast $(P$. grisea) and cucumber gray mold ( $B$. cinerea) were examined with the aid of High Throughput Screening. A testing solution was obtained by dissolving compounds in acetone to the desired concentration of $25 \mathrm{mg} / \mathrm{L}$. The testing solution and phytopathagen suspensions were added into a 96-well culture plate and the reaction mixture was maintained in the constant temperature chamber. After $24 \mathrm{~h}$ phytopathagen germination or growth, the fungicidal activity of the compound was assessed relative to phytopathagen germination or growth in control experiment. The level of fungicidal activity was reported in the range of 0 (no control) to $100 \%$ (complete control).

\subsubsection{Insecticidal and acaricidal activity bioassay}

All of evaluations were performed triplicates for each concentration and used the procedures in the references. ${ }^{[33]}$ All bioassays were carried out at $25{ }^{\circ} \mathrm{C}$ on the representative insects reared in the laboratory. The assessments were made on a dead-to-alive basis, and the mortality rates were corrected using Abbott's formula. The results were reported ranging from $0 \%$ (no activity) to $100 \%$ (totally killed).

The insecticidal activities of the target compounds against diamondback moth ( $P$. xylostella) were tested by the leaf-dip method. Leaf disks $(6 \mathrm{~cm} \times 2 \mathrm{~cm})$ were cut from fresh cabbage leaves and then dipped into the test solution for $3 \mathrm{~s}$. After air-drying, the treated leaf disks were placed individually into glass tubes. Each treated leaf disk was infected with seven third-instar diamondback moth larvae. The percentages of mortalities were assessed $4 \mathrm{~d}$ after treatment. The control experiment was carried out using the 
leaves treated with the solution of water and $N, N$-dimethylformamide (DMF).

The insecticidal activities of the target compounds against oriental armyworm (M. separata) were evaluated by foliar application. Corn leaves were placed on moistened pieces of filter paper on Petri dishes. The leaves were then sprayed with the test solution and allowed to air-dry. The dishes were infested with 10 fourth-instar oriental armyworm larvae. The percentage of mortality were evaluated $4 \mathrm{~d}$ after treatment.

The insecticidal activities of the target compounds against Peach Aphid (M. persicae) were evaluated by foliar application. Corn leaves were placed on moistened pieces of filter paper in Petri dishes and leaf disks $(3 \mathrm{~cm} \times 3 \mathrm{~cm})$ were cut from the above leaf. Then the corn leaves were sprayed with the target compound $(0.5 \mathrm{~mL})$ and then placed in a humidity room $(60 \% \sim 70 \%)$ at $25{ }^{\circ} \mathrm{C}$ for visual observation. The percentage of mortality was assessed $2 \mathrm{~d}$ after treatment.

The acaricidal activities of the target compounds against larvae of spider mite (T. cinnabarinus) were evaluated. The mite-egg-infested leaves (eggs that were laid on the same day) were maintained at $25{ }^{\circ} \mathrm{C}$ for $4 \mathrm{~d}$. Then they were placed on leaves of tested plants as above. After 1 day, the larvae were hatched and moved to the fresh leaves. Each leaf was about $60 \sim 100$ mites. The leaf was cut and dipped into the test solution for $3 \mathrm{~s}$, the excess liquid was removed, and then the leaf was placed in a tube $(10 \mathrm{~cm}$ inner diameter) lined with a piece of filter paper. The percentage of mortality was evaluated $4 \mathrm{~d}$ after treatment.

Supporting Information NMR and HR-ESI mass spectra of target compounds, crystal structure data of 7a. The Supporting Information is available free of charge via the Internet at http://sioc-journal.cn.

\section{References}

[1] Schobert, R.; Schlenk, A. Bioorg. Med. Chem. 2008, 16, 4203.

[2] Reddy, D. K.; Shekhar, V.; Prabhakar, P.; Babu, D. C.; Ramesh, D.; Siddhardha, B.; Murthy, U. S. N.; Venkateswarlu, Y. Bioorg. Med. Chem. Lett. 2011, 21, 997.

[3] Mo, X.; Li, Q.; Ju, J. RSC Adv. 2014, 4, 50566.

[4] Liu, Y.; Cui, Z.; Zhao, H.; Li, Y.; Gu, Y.; Wang, Q. J. Heterocycl. Chem. 2014, 51, E209.

[5] Zhang, C. L.; Chatterjee, S.; Stein, U.; Heinemann, U. Pharmacology 1992, 345, 85 .

[6] Liu, Z.; Lei, Q.; Li, Y.; Xiong, L.; Song, H.; Wang, Q. J. Agric. Food Chem. 2011, 59, 12543.

[7] Bretschneider, T.; Fischer, R.; Benet-Buchholz, J. Pflan-
zenschutz-Nachr. Bayer 2005, 58, 307.

[8] Fischer, R.; Benet-Buchholz, J. Pflanzenschutz-Nachr. Bayer 2002, 55,2 .

[9] Muehlebach, M.; Boeger, M.; Cederbaum, F.; Cornes, D.; Friedmann, A. A.; Glock, J.; Niderman, T.; Stoller, A.; Wagner, T. Bioorg. Med. Chem. 2009, 17, 4241.

[10] Zhao, J. H.; Ji, M. H.; Xu, H. X.; Cheng, J. L.; Zhu, G. N. Chin. Chem. Lett. 2009, 20, 1307.

[11] Patel, J. B.; Desai, V. A. Int. J. Drug Des. Discovery 2011, 2, 393.

[12] Ivanna, S.; Dmytro, A.; Ewa, S.; Katarzyna, K. K.; Borys, Z.; Olexandr, V.; Andrezj, G.; Roman, L. Bioorg. Med. Chem. 2010, 18, 5090.

[13] Tang, B.; Yang, M. Y.; Zhao, Y.; Kong, L. Q.; Wang, W. W.; Wang, M. A. Molecules 2015, 20, 13740.

[14] Zhao, Y.; Tang, B.; Guan, A. Y.; Wang, W. W.; Zhang, Z. H.; Wang, M. A. Synthesis 2017, 49, 4663.

[15] Tang, B.; Guan, A. Y.; Zhao, Y.; Jiang, J. Z.; Wang, M. A.; Zhou, L. G. Chin. J. Chem. 2017, 35, 1133.

[16] Guan, A. Y.; Zhao, Y.; Wang, W. W.; Liu, X. L.; Wang, M. A. Chin. J. Org. Chem. 2018, 38, 2767 (in Chinese).

(关爱莹, 赵宇, 王卫伟, 刘金歪, 王明安, 有机化学, 2018, 38, 2767.)

[17] Zhao, Y.; Tang, B.; Liu, X. L.; Li, W. Z.; Huang, M. Y.; Wang, M. A. Chin. J. Org. Chem. 2017, 37, 957 (in Chinese). (赵宇, 汤博, 刘釒否, 李婉祯, 黄铭一, 王明安, 有机化学, 2017, 37, 957.)

[18] Liu, Y. X.; Cui, Z. P.; Li, Y. H.; Gu, Y. C.; Wang, Q. M. J. Heterocycl. Chem. 2014, 51, E197.

[19] Hu, Y.; Wang, J.; Lu, A.; Yang, C. Bioorg. Med. Chem. Lett. 2014, 24, 3772 .

[20] Zhang, L. Z.; Ren, Z. J.; Lu, A. M.; Zhao, Z.; Yang, C. L. Chem. Res. Chin. Univ. 2015, 31, 228.

[21] Asif, M. Int. J. Adv. Chem. 2014, 2, 85.

[22] Sersin, F.; Gregan, F.; Pesco, M.; Dvoranova, D.; Donovalova, J. Molecules 2015, 20, 14139.

[23] Geng, P. F.; Liu, X. Q.; Zhao, T. Q.; Wang, C. C.; Li, Z. H.; Zhang, J.; Wei, H. M.; Hu, B.; Ma, L. Y.; Liu, H. M. Eur. J. Med. Chem. 2018, 146, 147.

[24] Addor, R. W.; Kuhn, D. G.; Wright, D. P. EP 228564, 1987.

[25] Zhang, X. N.; Li, Y. F.; Ni, J. P. Pesticide 2003, 18.

[26] Wing, K. D.; Richard, A.; Carlson, G. R. Science 1988, 241, 470.

[27] Retnakaran, A.; Hiruma, K.; Palli, S. R.; Riddiford, L. M. Insect Biochem. Mol. Biol. 1995, 25, 109.

[28] Kusano, H.; Oga, Y.; Ishida, N.; Satsuki, M.; Suga, S. JP 2001294585, 2001

[29] Wei, R.; Liu, Y.; Liang, Y. Chin. J. Org. Chem. 2009, 29, 476 (in Chinese).

(魏荣宝, 刘洋, 梁娅, 有机化学, 2009, 29, 476.)

[30] Yan, T.; Yu, S. J.; Liu, P. F.; Liu, Z.; Wang, B. L.; Xiong, L. X.; Li, Z. M. Chin. J. Chem. 2012, 30, 919.

[31] Guan, A. Y.; Wang, M. A.; Yang, J. L.; Wang, L. Z.; Xie, Y.; Chen, W.; Lan, J.; Liu, C. L. J. Agric. Food Chem. 2017, 65, 10829.

[32] Guan, A. Y.; Liu, C. L.; Chen, W.; Yang, F.; Xie, Y.; Zhang, J. B.; Li, Z. N.; Wang, M. A. J. Agric. Food Chem. 2017, 65, 1272.

[33] Yang, J. C.; Li, M.; Wu, Q.; Liu, C. L.; Chang, X. H. Bioorg. Med. Chem. 2016, 24, 383. 